Document downloaded from:

http://hdl.handle.net/10251/54466

This paper must be cited as:

Sanchez Fandiño, JA.; Muñoz Muñoz, P. (2015). Analysis of System Imperfections in a Photonics-Assisted Instantaneous Frequency Measurement Receiver Based on a DualSideband Suppressed-Carrier Modulation. Journal of Lightwave Technology. 33(2):293-303. doi:10.1109/JLT.2014.2378331.

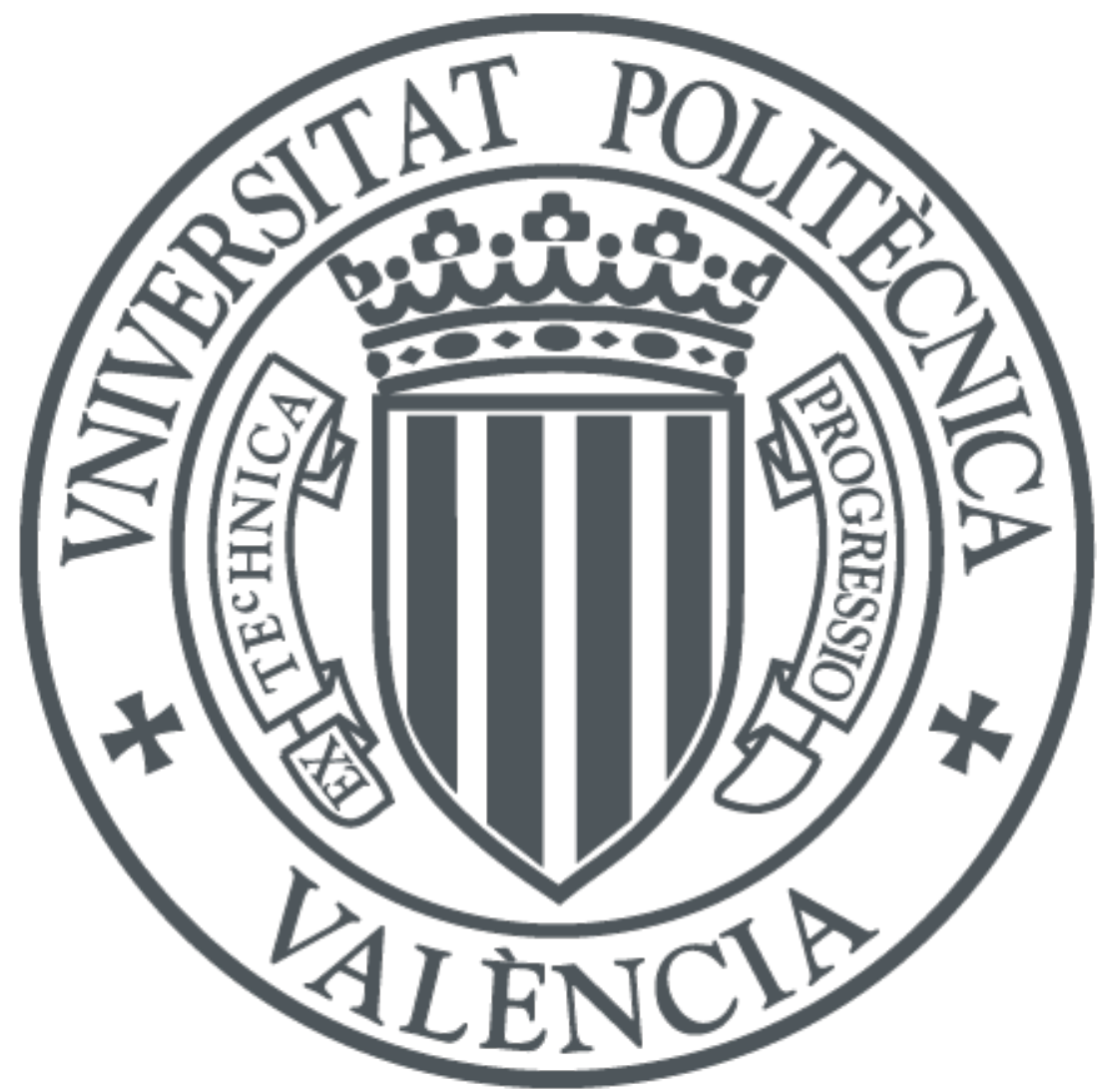

The final publication is available at

http://dx.doi.org/10.1109/JLT.2014.2378331

Copyright Institute of Electrical and Electronics Engineers (IEEE)

Additional Information 


\title{
Analysis of System Imperfections in a Photonics-Assisted Instantaneous Frequency Measurement Receiver Based on a Dual-Sideband Suppressed-Carrier Modulation
}

\author{
Javier S. Fandiño and P. Muñoz, Senior Member, IEEE, Member, OSA
}

\begin{abstract}
Instantaneous frequency measurement receivers are a well-established technology that is used for the ultrafast characterization of pulsed microwave signals over a broad bandwidth. Recently, numerous photonic approaches to instantaneous frequency measurement (IFM) have been proposed and experimentally demonstrated, with the ultimate aim of leveraging the benefits of optical technology to improve the performance of already existent electronic solutions. Despite the numerous results, not so much attention has been paid so far to understand the subtle implications that system imperfections can have on realistic photonics-based IFM receivers. Here, we focus our attention in one of the most promising among these IFM techniques, which is based in optical power monitoring of a dual-sideband suppressedcarrier modulation after a Mach-Zehnder interferometer (MZI) filter. We develop a time domain model for the rigorous analysis of all major optical and electrical effects, including amplitude imbalance and phase errors in the modulator and the MZI, as well as on-pulse RF phase/frequency modulation. Simulations are then used to illustrate the substantial effect that a non-perfectly suppressed optical carrier can have on system performance. More importantly, it is shown that in a non-ideal situation the system amplitude comparison function critically depends with input RF power, thus greatly limiting the dynamic range of the photonicsbased receiver. Some approaches to solve these issues are also discussed.
\end{abstract}

Index Terms-Frequency measurement, Integrated optoelectronics, Microwave measurements, Optical planar waveguides, Optical waveguide filters.

\section{INTRODUCTION}

D ETECTING and characterizing electromagnetic activity in nearly real-time is of critical importance for many modern electronic warfare (EW) systems [1], [2]. For example, radar warning receivers (RWR) installed in military aircrafts continuously intercept and process radar signals during tactical operations. Trains of detected RF pulses are usually classified according to their physical characteristics, and these are then used to estimate the type and origin of the received waveforms. By doing so, potential threats can be identified (such as hostile

This work was supported by projects TEC2010-21337 (ATOMIC), FEDER UPVOV10-3E-492, FEDER UPVOV08-3E-008, TEC2013-42332-P (PIC4ESP) and PROMETEO 2013/012. The work of J. S. Fandiño was supported by grant FPU-2010 (ref: AP2010-1595).

The authors are with the Optical and Quantum Communications Group (OQCG), ITEAM Research Institute, Universitat Politècnica de València, València, Spain (email: jasanfan@iteam.upv.es).

Copyright (c)2013 IEEE. Personal use of this material is permitted. However, permission to use this material for any other purposes must be obtained from the IEEE by sending a request to pubs-permissions@ieee.org. surveillance radars and radar guided weapons), and countermeasures can be either manually or automatically taken, if needed.

Due to the wide range of existent radar equipment, RWR must be able to perform pulse measurements over a very broad bandwidth (typically tens of $\mathrm{GHz}$ ) while still offering a quasi-instantaneous response. These stringent requirements are typically met by means of dedicated instantaneous frequency measurement (IFM) subsystems [3], [4]. Thanks to their special architecture, which usually combines a sophisticated mix of high-speed digital and analog electronics, IFM receivers can be used to estimate a wide range of RF pulse parameters with sub-microsecond response times, including: Center frequency $\left(f_{R F}\right)$, pulse width $(\mathrm{PW})$, pulse amplitude (PA) and pulse repetition frequency (PRF). Furthermore, since IFM systems directly measure the instantaneous frequency of the RF signal, on-pulse phase/frequency modulation can also be detected.

While electronic solutions are well established in the EW and signals intelligence (SIGINT) markets, the past few years have seen a remarkable increase in the proposal and development of photonic approaches to IFM [5]-[20]. Similarly to the well-known field of microwave photonics, which seeks to take advantage of the unparalleled benefits that optical technology can bring to microwave applications, all these techniques are aimed at improving the performance of current electronicsbased IFM products. In fact, the exploding telecom growth has imparted significant momentum in the development of different photonic integration platforms, which have already reached considerable levels of maturity in terms of functionality and performance of their basic building blocks [21]-[23]. By exploiting the benefits of these technologies, integrated, light-based systems hold the promise for potentially low cost, compact footprint and high-bandwidth IFM equipment. Among all the reported photonic approaches to IFM, those based on detection and processing of optical powers stand out as the most promising for a near and short term practical implementation [6], [8], [9], [11], [12], [14], [15], [19], [20], [24]. These avoid the need of microwave components and circuits (such as detection logarithmic video amplifiers), and only require the use of high sensitivity (i.e, low dark current) photodiodes and high dynamic range transimpedance amplifiers.

So far, most reported results have focused on proof-of- 


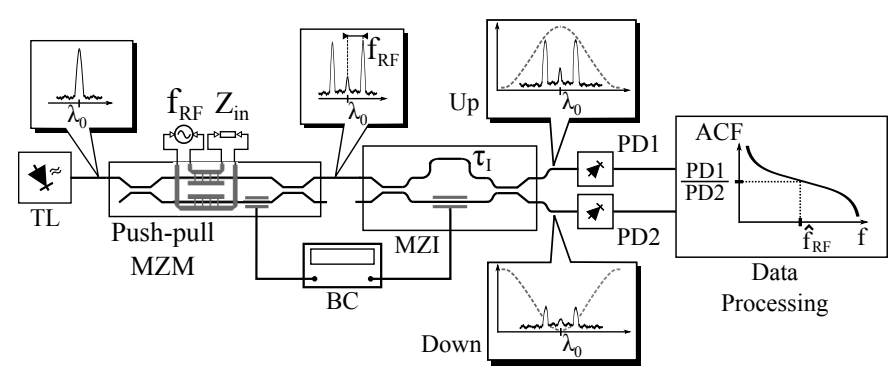

Fig. 1. Schematic diagram of the photonic IFM system under analysis. TL: Tunable laser. MZM: Mach-Zehnder Modulator. MZI: Mach-Zehnder Interferometer. BC: Bias Controller. PD: Photodetector. ACF: Amplitude Comparison Function.

principle experimental demonstrations, with little in-depth discussions on the subtleties and trade-offs associated with imperfect system operation. Besides, just continuous wave (CW), frequency domain analyses under highly ideal conditions have been provided in order to exemplify the main working principle behind each technique, as well as to provide a theoretical basis for the experimental data. This approach has two important shortcomings. First, frequency domain analyses are only able to compute the response of an IFM system under $\mathrm{CW}$ operation. This means that they can not capture temporal trade-offs, which are of great importance in practical systems where very short (and possibly chirped) RF pulses need to be measured. Second, idealistic theoretical analyses often neglect key effects that can impose severe performance constraints. For example, in one of our previous works we pointed out to the fact that the presence of a residual optical carrier in the modulator output can induce a significant bias error on the estimated central frequency of the received signal, and that this error is in fact dependent on input RF power [24]. Since residual optical carrier power arises from amplitude imbalance and phase errors in the modulator, it is obvious then that their impact on system performance needs to be carefully considered. A notable exception is a recent work by Harmon et al. [25], where a model for analog photonic links that can be applied to some IFM approaches is presented. This model accounts for some realistic effects like amplitude imbalance in the Mach-Zehnder interferometer (MZI) as well as bias phase errors in the modulator and the MZI. However, other important parameters (such as modulator amplitude imbalance and photodiodes dark currents) are not included, and only $\mathrm{CW}$ operation is considered.

Here, and to the best of our knowledge, the first timedomain-based theoretical analysis of an already reported IFM technique based on a double-sideband suppressedcarrier (DSB-SC) optical modulation and a MZI filter is presented [12]. We focused on this particular architecture because, contrary to similar techniques based on more complex filter structures [24], an MZI only requires a single bias electrode, which eliminates the need of complex control electronics for the continuous adjustment of the filter transfer function. This makes this architecture particularly suited for its implementation as an application specific photonic integrated circuit (ASPIC) with current state-of-the-art technology [26].
Furthermore, the simplicity of this filter naturally lends itself to be described by closed-form formulas, helping to better ascertain its intrinsic limitations, as well as to perform calibration corrections after appropriate measurements. Our analysis allows to compute the impact of amplitude imbalance and phase errors in both the Mach-Zehnder modulator (MZM) and the MZI, as well as the effect of temporal phase/frequency modulation in an incoming RF pulse, among other impairments (such as insertion losses and non-zero dark currents). Thus, it can serve as a powerful analysis and design tool for the development of IFM systems based on this particular technique, as well as for better understanding experimental data. Moreover, the results of our analysis show that great attention must be paid to the dependence of system performance with input RF power, since it can be significantly degraded when considering realistic parameter values on the photonic system. This is an important consideration that must be addressed with care when comparing photonics-based IFM systems with their electronic counterparts, which typically boast outstanding dynamic range specifications.

This work is organized as follows: First, a short description of the operation principle behind the analyzed IFM technique is presented, followed by the main assumptions and formulas of the time domain analysis. Second, some temporal trade-offs that follow from these equations are discussed. Third, numerical simulations are performed to evaluate the relative impact of each considered parameter on system performance. Fourth, realistic values extracted from state-of-the-art components are employed to illustrate the significant effect of input RF power in the frequency bias error. Finally, possible techniques for mitigating this effect are proposed along with a summary of the main results and conclusions.

\section{IFM THEORY}

\section{A. Operation principle}

Before going into a detailed theoretical description, it is illustrative to first review the basic idea behind this particular photonic approach to IFM. For that purpose, it is best to resort to a simplified frequency domain model, which gives a quick and visual interpretation of the underlying mechanism. The readers are nevertheless referred to the original work by Zou and coworkers for a more detailed explanation [12].

A system diagram of the technique can be seen in Fig. 1. It can be briefly described as follows. First, a CW laser source is introduced into a push-pull MZM, whose bias has been previously set at the minimum transmission point. The MZM is being fed by a continuous RF tone of unknown frequency $\left(f_{R F}\right)$ and amplitude $\left(A_{R F}\right)$. Under ideal circumstances (no amplitude imbalance and bias phase error), the MZM bias forces the optical carrier to perfectly interfere destructively with itself at the output of the interferometer, self-cancelling out. If the input RF power is low, then mainly the first order sidebands are present in the signal power spectrum, creating what is commonly known as a DSB-SC optical modulation. Finally, the signal is introduced into a MZI whose maximum/minimum transmission point (upper and lower branch, respectively) has been set to match the laser 


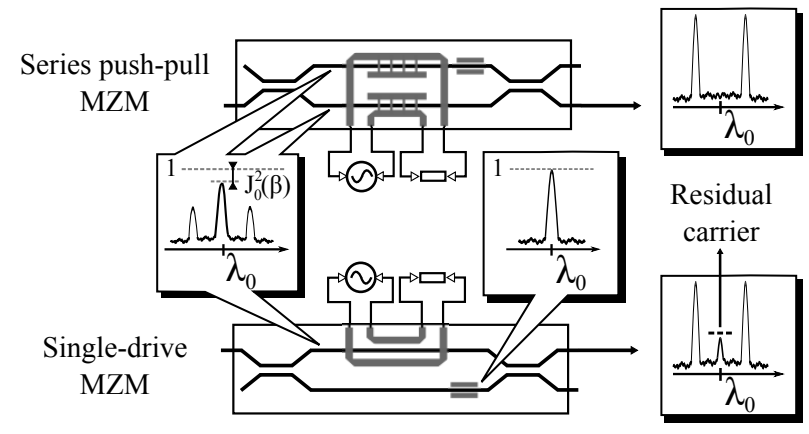

Fig. 2. Difference between single-drive and series push-pull operation in a MZM. Note that in the former case the carrier amplitude in each arm is different, while in the latter it remains balanced since the modulation process affects both arms equally.

central wavelength. Because of the complimentary nature of the spectral response of the MZI outputs, the first-order optical sidebands experience different levels of optical attenuation at each output. Finally, the powers are measured with two photodiodes (PD1 and PD2 in Fig. 1). Their ratio, also known as Amplitude Comparison Function (ACF), is only dependent on the spectral shape of the filter (which either has been previously characterized or is known by design), and the RF modulation frequency. As a result, an estimation $\left(\hat{f_{R F}}\right)$ of the unknown RF frequency can be found by just measuring two DC photocurrents, calculating their ratio and then computing the mathematical operation below:

$$
\hat{f_{R F}}=\frac{\mathrm{FSR}}{2 \pi} \arccos \left( \pm \frac{1}{\gamma} \frac{(\mathrm{ACF}-1)}{(\mathrm{ACF}+1)}\right)
$$

where FSR stands for the Free Spectral Range of the MZI, $\gamma$ is the measured peak-to-notch optical power ratio of the MZI transfer function (see [12]) and ACF stands for the measured DC photocurrent ratio (PD1 divided by PD2). Notice that there are two possible solutions ( + and - ), depending on which definition of ratio is chosen. If the ACF is defined as PD1/PD2, then the positive solution must be computed, and viceversa.

As a final remark, note that due to the non-injective properties of the $\arccos ()$ function, only frequency values between 0 and FSR/2 can be recovered without ambiguity. Besides, the MZI transfer function must be well known (FSR and $\gamma$ ) in order to avoid frequency errors during the estimation process. These errors arise due the fact that different input frequency estimations $\left(\hat{f_{R F}}\right)$ are obtained for different values of $\gamma$ and FSR even if the same photocurrent ratio is measured. Thus, either these variations (caused due to manufacturing tolerances) are kept low in order to set an upper limit on the frequency error, or hardware-embedded calibration curves are employed to compensate for systematic errors, of course at the expense of an increase in cost and computational complexity.

\section{B. Time domain analysis}

We now start by assuming that at the input of our system there is an arbitrary, real, passband microwave signal of the form

$$
V_{R F}(t)=A_{R F} p(t) \cos \left(2 \pi f_{R F} t+\phi_{R F}(t)\right)
$$

where $A_{R F}$ is the amplitude, $p(t)$ is a normalized pulse shape (that is, $p(t) \leq 1$ ), $f_{R F}$ is the central frequency of the RF signal and $\phi_{R F}(t)$ accounts for a possible on-pulse phase/frequency modulation (such as those of chirped radars).

This unknown waveform, of which we wish to estimate its instantaneous frequency (defined as $\left.f(t)=f_{R F}+(1 / 2 \pi) \partial \phi_{R F}(t) / \partial t\right)$, is then employed to modulate a $\mathrm{CW}$ laser. From now on, optical signals will be described by low-pass, analytic (i.e. complex-valued) functions of time. These will be written with an upper tilde, in order to distinguish them from the real-valued signals associated with input RF pulses and detected photocurrents. As an example, a CW laser can be simply described as $\tilde{E}_{o}(t)=\sqrt{P_{o}} e^{j \phi_{o}(t)}$, where $P_{o}$ is the output power and $\phi_{o}(t)$ models the laser phase noise. For the sake of simplicity, phase noise will not be considered $\left(\phi_{o}(t)=0\right)$.

It has been stated in the previous section that the purpose of the MZM is to encode the microwave signal information into a DSB-SC modulation, from which its instantaneous frequency can be estimated after appropriate optical and electrical processing. As it will be shown later, the ability of the MZM to reduce the power of the optical carrier is crucial for obtaining a RF power independent estimation of the instantaneous frequency. Due to its critical role, appropriate modelling of possible non-ideal effects is mandatory. In fact, not only non-ideal parameters can affect the quality of the DSB-SC modulation, but also the topology of the MZM itself. As shown in the system diagram of Fig. 2, it is important for the modulation process to take place inside a push-pull MZM. The main reason is that by differentially driving both arms of the MZM (push-pull operation) one can ideally achieve perfect carrier suppression. On the contrary, if only one arm is modulated, then the powers of the optical carriers travelling on each branch are no longer different (due to energy conservation, a certain fraction of the carrier power in the modulating arm is diverted to create the sidebands). Since they have no equal amplitudes, the two optical carriers can no longer perfectly interfere destructively, even if they are exactly out of phase and no power imbalance exists in the modulator branches. Thus, differentially driving the MZM is of fundamental importance for achieving perfect carrier suppression. For that purpose, either a series push-pull architecture or a dual-drive MZM with an external $180^{\circ} \mathrm{RF}$ hybrid can be employed. The latter implies that external RF imperfections in the hybrid (power imbalance and phase errors) must be additionally accounted for, whereas the former avoids the use of external, expensive RF circuits, reducing costs and simplifying the set-up. This implies that series push-pull MZMs are the optimal choice for this particular application. Fortunately, this is one of the most typical topologies found in both $\mathrm{LiNbO} 3$ (x-cut) and InP modulators, so off-the-shelf high-performance components are readily available [27]-[29].

We will subsequently assume that a series push-pull MZM is being employed, which can be characterized by the following parameters (see Fig. 3):

- $\phi_{M}$ : Modulator bias phase.

- $\beta_{o}(t)$ : Arbitrary time-varying phase modulation index due to the RF signal (this is, $(1 / 2)\left(\pi / V_{\pi}^{A C}\right) V_{R F}(t)$ ). Here, 


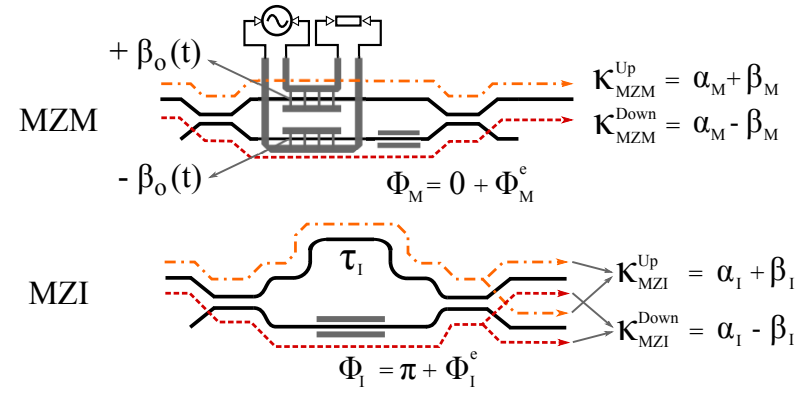

Fig. 3. MZI and MZM parameters considered in the theoretical analysis. In the MZI case, the second coupler is assumed to be perfectly symmetric so only 2 parameters are needed to model the imbalance.

$V_{\pi}^{A C}$ stands for the dynamic voltage required in one arm of the MZM to induce a $\pi$ phase shift on the travelling optical wave, and the $1 / 2$ factor is due to the push-pull operation. Also notice that $V_{\pi}^{A C}$ normally depends on the frequency of the modulating signal. It thus accounts for all those physical processes that can affect modulation efficiency, such as RF losses, as well as impedance and velocity mismatch.

- $\kappa_{M Z M}^{U p}$ and $\kappa_{M Z M}^{D o w n}$ : Electric field attenuation factors as seen by the laser field when it propagates trough the upper/lower arms of the interferometer, respectively (see Fig. 3). These only account for losses and imbalance, and thus exclude the phase shifts in the optical couplers.

In practice $\kappa_{M Z M}^{U p}$ and $\kappa_{M Z M}^{D o w n}$ can not be directly measured. Typically, only the optical transmission curve versus DC voltage is available. This curve is indeed defined by the maximum and minimum optical power transmission points ( $T_{\max }$ and $\left.T_{\min }\right)$, as well as by the DC bias voltage periodicity. We can however relate the two electric field attenuation factors to the measured MZM transmission curve by noting that $T_{\max }=\left(\kappa_{M Z M}^{U p}+\kappa_{M Z M}^{\text {Down }}\right)^{2}$ and $T_{\text {min }}=\left(\kappa_{M Z M}^{U p}-\kappa_{M Z M}^{\text {Down }}\right)^{2}$. In order to simplify the notation, we will rewrite these factors as

$$
\begin{aligned}
\kappa_{M Z M}^{U p} & =\alpha_{M}+\beta_{M} \\
\kappa_{M Z M}^{D o w n} & =\alpha_{M}-\beta_{M}
\end{aligned}
$$

where $\alpha_{M}$ and $\beta_{M}$ are defined as

$$
\begin{aligned}
\alpha_{M} & =\frac{\kappa_{M Z M}^{U p}+\kappa_{M Z M}^{D o w n}}{2} \\
\beta_{M} & =\frac{\kappa_{M Z M}^{U p}-\kappa_{M Z M}^{D o w n}}{2}
\end{aligned}
$$

Now, it is easy to see that

$$
T_{\max }=4 \alpha_{M}^{2} \quad T_{\min }=4 \beta_{M}^{2}
$$

which are directly related to the optical extinction ratio (ER) and insertion losses (IL) of the device, in linear units:

$$
\begin{gathered}
\mathrm{ER}_{M}=\frac{T_{\text {max }}}{T_{\min }}=\frac{\alpha_{M}^{2}}{\beta_{M}^{2}} \\
\mathrm{IL}_{M}=T_{\text {max }}=4 \alpha_{M}^{2}
\end{gathered}
$$

Thus, by just measuring both the ER and IL of the MZM, $\alpha_{M}$ and $\beta_{M}$ can be computed through Eqs. (8) and (9). Ideally, $\mathrm{ER}=\infty$ and $\mathrm{IL}=1$, so $\alpha_{M}=1 / 2$ and $\beta_{M}=0$. Also note that there are two possible values of $\beta_{M}$ (either positive or negative) that result in the same ER. This is due to the fact that it is in general not possible to know which arm is causing more attenuation than the other.

While the electric field attenuation factors are essentially constant with time, the modulator bias phase will slowly drift as a result of changes in the operating conditions of the system, including temperature changes, ageing and static electrical charge accumulation. As a consequence, the bias phase of the modulator needs to be carefully monitored and adjusted. Here, we assume that an automatic bias control circuit is keeping the upper output at the minimum transmission point except for a possible phase error $\left(\phi_{M}=0+\phi_{M}^{e}\right)$, as shown in Fig. 3. This phase error arises from possible limitations in the technique employed by the bias controller, which typically are in the order of $\pm 1^{\circ}$ [30]. In that case, the electric field at the output of the MZM can be finally expressed as

$$
\begin{aligned}
\tilde{E}_{M Z M}(t)= & \tilde{E}_{o}(t)\left[\left(\alpha_{M}+\beta_{M}\right) e^{j \beta_{o}(t)}\right. \\
& \left.-\left(\alpha_{M}-\beta_{M}\right) e^{j \phi_{M}^{e}} e^{-j \beta_{o}(t)}\right]
\end{aligned}
$$

The non-ideal DSB-SC modulation is then introduced into an MZI. Making similar assumptions as in the previous case, we have that the MZI can be described by the following parameters:

- $\tau_{I}$ : Time delay difference between the upper and lower arms of the interferometer, which is related to the FSR of the filter by FSR $=\tau_{I}^{-1}$. It is assumed that the dispersion of the interferometer delay line is first-order only, since second-order effects (pulse broadening and distortion) are negligible given the low propagation distances of practical integrated devices.

- $\kappa_{M Z I}^{U p}$ and $\kappa_{M Z I}^{D o w n}$ : Real-valued constants that model the electric field attenuation as seen by the laser when it propagates through the branches of the MZI. As explained before, the related parameters $\alpha_{I}$ and $\beta_{I}$ can be computed by first measuring $\mathrm{ER}_{I}$ and $\mathrm{IL}_{I}$, and then using Eqs. (8) and (9). Please note that, if the output coupler is not perfectly balanced, the MZI would need to be modelled using 4 different electric field attenuation constants. These would correspond to the 4 different optical paths that the light follows within the device before it recombines at the output ports. In that case, both outputs of the MZI would need to be individually characterized, yielding different insertion losses and extinction ratios for each port. In most practical devices, however, the imbalance of the MZI is mainly due to the extra loss caused by the longer propagation distance in the upper arm (which can be significant if the FSR is small), and not due to the imbalance of the output coupler. In fact, integrated MZIs typically use MMIs as output couplers, which are well-known for their inherent robustness to manufacturing deviations. If properly designed, these devices show outstanding performance (see for example [31]), and thus their contribution to 


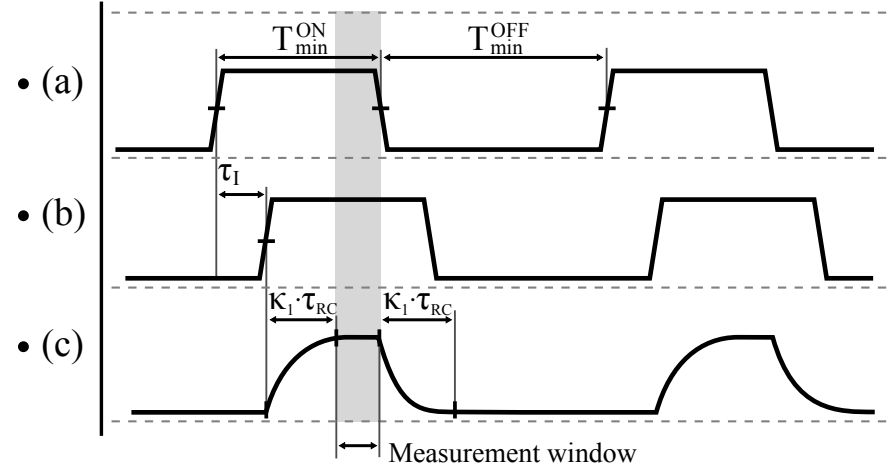

Fig. 4. Temporal evolution of signals inside the IFM system. (a) Input RF pulse. (b) Delayed RF pulse after propagation through the long MZI arm (c) Resulting low-speed photocurrent term containing the information of the instantaneous frequency.

the overall imbalance can be considered of second order. For simplicity, we will assume that this asymmetry in the output coupler is negligible, so both outputs can be characterized using just two parameters. Nevertheless, these extra variables could be easily incorporated into the model if they were required.

- $\phi_{I}$ : Interferometer bias phase. It is also assumed to be continuously adjusted so that the wavelength of maximum (minimum) transmission when coming out by the upper (lower) output matches the emission wavelength of the laser. That is, $\phi_{I}=\pi+\phi_{I}^{e}$, where $\phi_{I}^{e}$ is a possible phase error due to an imperfect control of the bias point.

As a result, the electric field at the upper/lower output of the MZI can be written down as

$$
\begin{aligned}
\tilde{E}_{M Z I}^{U p}(t)= & {\left[\left(\alpha_{I}+\beta_{I}\right) \tilde{E}_{M Z M}\left(t-\tau_{I}\right)\right.} \\
& \left.+\left(\alpha_{I}-\beta_{I}\right) \tilde{E}_{M Z M}(t) e^{j \phi_{I}^{e}}\right] \\
\tilde{E}_{M Z I}^{\text {Down }}(t)= & (-j)\left[\left(\alpha_{I}+\beta_{I}\right) \tilde{E}_{M Z M}\left(t-\tau_{I}\right)\right. \\
& \left.-\left(\alpha_{I}-\beta_{I}\right) \tilde{E}_{M Z M}(t) e^{j \phi_{I}^{e}}\right]
\end{aligned}
$$

Finally, both optical signals are detected in a couple of low-speed, high sensitivity photodiodes (PD1 and PD2). The photodetection process will be described as a current source whose output linearly depends with the input, instantaneous, time-averaged optical power $\left(\overline{P_{o}}(t)=\frac{1}{T} \int_{t-T / 2}^{t+T / 2}|\tilde{E}(t)|^{2} d t\right)$ impinging upon the pin junction. This averaging time is assumed to be much higher than the period of the optical signal, but lower than the temporal variations of the RF pulse amplitude $(p(t))$, so that

$$
i(t) \simeq \mathcal{R} \int_{-\infty}^{t}|\tilde{E}(s)|^{2} h(t-s) d s+I_{\text {Dark }}
$$

where $\mathcal{R}$ stands for the photodiode's responsivity, $I_{\text {Dark }}$ is the photodiode's dark current, and $h(t)$ is the current impulse response of the whole output circuit. Since we are interested in low-speed operation (small bandwidth photodiodes), $h(t)$ can be modelled to first-order as a simple RC circuit: $H(\omega)=(1+j \omega R C)^{-1}$. Its $3 \mathrm{~dB} \mathrm{RF}$ bandwidth is given by $f_{3 d B}=(2 \pi R C)^{-1}$, where $R$ is the total output resistance (series + load) and $C$ is mainly dominated by the junction capacitance.
Substituting Eq. (10) into both (11) and (12) yields the electric fields at the input of the photodiodes. Their time-averaged optical power is easily computed as $\overline{P_{o}}(t)=|\tilde{E}(t)|^{2}=\tilde{E}(t) \tilde{E}^{*}(t)$, from which output photocurrents can be finally obtained through (13). Alternatively, this last step can also be done by eliminating those RF beating terms which are not below the cut-off frequency of the photodiode.

\section{Temporal trade-offs}

Now, and prior to developing on the exact formulas of the detected photocurrents, it is instructive to have a look at their general form. Due to the multiplication (or beating) among all possible combinations of the delayed and non-delayed modulated electric fields, these electrical signals are made of a linear combination of quasi-DC (low speed) and RF (highspeed) terms. However, it is important to note that, out of all beating terms, only those formed by a multiplication between a delayed and non-delayed signal contain information about the instantaneous frequency that is to be measured. To see this, we can write down the general formula for one of these beating terms as

$$
\begin{aligned}
i^{\prime}(t) & \propto \Re\left\{e^{j \beta_{o}(t)} e^{-j \beta_{o}\left(t-\tau_{I}\right)}\right\}= \\
& =\Re\left\{e^{j z(t) \cos (\theta(t))} e^{-j z\left(t-\tau_{I}\right) \cos \left(\theta\left(t-\tau_{I}\right)\right)}\right\}
\end{aligned}
$$

where by simple identification $z(t)=(1 / 2)\left(\pi / V_{\pi}^{A C}\right) p(t) A_{R F}, \theta(t)=2 \pi f_{R F} t+\phi_{R F}(t)$ and $\Re\{\}$ stands for the real part of a complex number.

After employing the well-known Jacobi-Anger expansion $\left(e^{j z(t) \cos (\theta(t))}=\sum_{n=-\infty}^{+\infty} j^{n} J_{n}(z(t)) e^{j n \theta(t)}\right)$ and assuming that the photodiode $\mathrm{RC}$ circuit perfectly filters all high-order $\mathrm{RF}$ terms, this equation can be reformulated as

$$
\begin{aligned}
i^{\prime}(t) & \propto J_{o}(z(t)) J_{o}\left(z\left(t-\tau_{I}\right)\right)+ \\
& +2 \sum_{n=+1}^{+\infty} J_{n}(z(t)) J_{n}\left(z\left(t-\tau_{I}\right)\right) \cos \left(n\left(\theta(t)-\theta\left(t-\tau_{I}\right)\right)\right.
\end{aligned}
$$

Equation (15) has some important consequences. First, it tells us that the amplitude of the detected quasi-DC photocurrent is dependent on the first-order difference between time-delayed copies of the RF instantaneous phase $\left(2 \pi f_{R F} t+\right.$ $\left.\phi_{R F}(t)\right)$. Given that both $\tau_{I}$ and $z(t)$ are sufficiently small, this difference provides a good estimate $(\hat{f}(t))$ to the instantaneous frequency of the RF signal $(f(t))$ that we want to measure. This is,

$$
\begin{aligned}
\hat{f}(t) & =\frac{1}{2 \pi} \frac{\theta(t)-\theta\left(t-\tau_{I}\right)}{\tau_{I}}= \\
& =f_{R F}+\frac{1}{2 \pi} \frac{\phi_{R F}(t)-\phi_{R F}\left(t-\tau_{I}\right)}{\tau_{I}} \simeq \\
& \simeq f_{R F}+\frac{1}{2 \pi} \frac{\partial \phi_{R F}(t)}{\partial t}=f(t)
\end{aligned}
$$

Thus, the instantaneous frequency of the RF signal (and not only central frequency) can indeed be extracted by measuring the amplitude of the quasi-DC photocurrent. Ultimately, this means that chirped RF signals can be measured and characterized, provided that the variation time of the chirped signal 


$$
\begin{gathered}
I_{D C}=4\left(1+\frac{1}{\mathrm{ER}_{I}}\right)\left(1+\frac{1}{\mathrm{ER}_{M}}\right) \\
i_{\zeta}(\beta)=4\left(1+\frac{1}{\mathrm{ER}_{I}}\right)\left(1-\frac{1}{\mathrm{ER}_{M}}\right) \cos \left(\phi_{M}^{e}\right) J_{o}(2 \beta) \\
\sigma=4\left(1-\frac{1}{\mathrm{ER}_{I}}\right) \cos \left(\phi_{I}^{e}\right) \\
i_{\Delta}(\beta)=\left[\left(1-\cos \left(\phi_{M}^{e}\right)\right)+\frac{1}{\mathrm{ER}_{M}}\left(1+\cos \left(\phi_{M}^{e}\right)\right)\right] J_{o}^{2}(\beta) \\
i_{\Sigma}\left(\beta, f_{R F}, \phi_{R F}(t)\right)=2 \sum_{n=+1}^{+\infty}\left[\left(1-(-1)^{n} \cos \left(\phi_{M}^{e}\right)\right)+\frac{1}{\mathrm{ER}_{M}}\left(1+(-1)^{n} \cos \left(\phi_{M}^{e}\right)\right)\right] J_{n}^{2}(\beta) \cos \left(n\left[2 \pi f_{R F} \tau_{I}+\phi_{R F}(t)-\phi_{R F}\left(t-\tau_{I}\right)\right]\right)
\end{gathered}
$$

is slow enough to be detected by the low-pass response of the photodiodes.

Second, $z(t)$ (which is related to the amplitude of the RF waveform), needs to be kept low. Otherwise, contributions due to higher-order terms in the Bessel expansion will start to dominate, inducing an error in the estimated instantaneous frequency. This can be more easily seen by employing the frequency domain formalism mentioned before. When the input RF power is high enough, higher-order harmonics in the signal spectrum dominate over both the carrier and the first-order ones.

Third, the instantaneous frequency can not be obtained unless the temporal duration of the input RF pulse is at least longer than the interferometer time delay $\left(T_{\min } \geq \tau_{I}\right)$, as shown in Fig. 4. Otherwise, the product $J_{1}(z(t)) J_{1}\left(z\left(t-\tau_{I}\right)\right)$ will be zero and the instantaneous frequency of the signal will have no impact on the detected photocurrent. Last but not least, the finite response time of the photodiode implies that the steady-state value of the beating term is not reached until a certain time after the RF pulse enters the MZM. Settling times of RC circuits are typically specified as multiples $\left(\kappa_{1}\right)$ of the RC time constant $\left(\tau_{R C}=R C\right)$, where the exact value of $\kappa_{1}$ depends on the level of convergence to the final steady-state value specified in $\%$. As a consequence, a reliable estimation of $f(t)$ implies that the minimum duration of an RF pulse must satisfy

$$
T_{\min }^{O N} \geq \tau_{I}+\kappa_{1} \tau_{R C}
$$

where $\kappa_{1}$ is an arbitrary real constant (typically, $\kappa_{1} \geq 5$ for a convergence value better than $99 \%$ ).

Usually, $\tau_{I}$ is much lower than $\kappa_{1} \tau_{R C}$, so that $T_{\text {min }}^{O N} \simeq \kappa_{1} \tau_{R C}$. Also, since we are only interested in the quasi-DC beating terms, the photodiode $3 \mathrm{~dB}$ bandwidth must be much lower than the minimum instantaneous frequency $\left(f_{\text {min }}\right)$ in order to filter out all unwanted RF contributions. This is, $f_{3 d B}=\left(2 \pi \tau_{R C}\right)^{-1}=\kappa_{2} f_{\text {min }}$, being $\kappa_{2}$ an arbitrary constant much lower than 1 . Substituting these relations and defining $\kappa=\kappa_{1} /\left(2 \pi \kappa_{2}\right)$, we finally have that

$$
T_{\min }^{O N} \geq \frac{\kappa}{f_{\min }}
$$

Thus, it can be seen that there exists an intrinsic tradeoff between the minimum temporal width of an input pulse and the minimum measurable instantaneous frequency. As a consequence, quasi-DC instantaneous frequencies $\left(f_{\min } \rightarrow 0\right)$ can not be measured except for very long input RF pulses $\left(T_{\min } \rightarrow+\infty\right)$.

Using similar arguments, it is also easy to see that $T_{\min }^{O F F} \geq \kappa_{1} \tau_{R C}=T_{m i n}^{O N}$. As a result, an approximate upper limit on the maximum measurable pulse repetition frequency (PRF) of the input pulses is obtained:

$$
\mathrm{PRF}_{\max } \leq \frac{1}{2 T_{\min }^{O N}}=\frac{f_{\text {min }}}{2 \kappa}
$$

\section{Non-ideal Amplitude Comparison Function}

So far, just the general form of the detected low-speed photocurrents has been analyzed in order to get a deeper understanding of their intrinsic temporal trade-offs. Now, we turn to their exact expressions, which will allow us to compute the effect of each parameter on system performance. To do this, we will assume for simplicity that the amplitude of an input RF pulse has reached a steady state after a certain time (as seen in Fig. 4), so that $z(t)=\beta=(1 / 2)\left(\pi / V_{\pi}^{A C}\right) A_{R F}$. Appropriate substitutions and a considerable amount of algebra yield the following equations:

$$
\begin{gathered}
i^{\mathrm{Up}}=\eta\left(I_{D C}-i_{\zeta}(\beta)+\sigma\left[i_{\Delta}(\beta)+i_{\Sigma}\left(\beta, f_{R F}, \phi_{R F}(t)\right)\right]\right)+I_{\text {Dark }} \\
i^{\text {Down }}=\eta\left(I_{D C}-i_{\zeta}(\beta)-\sigma\left[i_{\Delta}(\beta)+i_{\Sigma}\left(\beta, f_{R F}, \phi_{R F}(t)\right)\right]\right)+I_{\text {Dark }}
\end{gathered}
$$

where $i^{\mathrm{Up}}$ and $i^{\text {Down }}$ are the detected quasi-DC photocurrents in the upper/lower photodiodes (PD1 and PD2, respectively), 
$\eta=\left(\mathrm{IL}_{M} \mathrm{IL}_{I} \mathcal{R} P_{o} / 16\right), I_{\text {Dark }}$ is the photodiode's dark current, and the definition of all the other terms can be found in Eqs. (22) to (26). Note that both extinction ratios $\left(\mathrm{ER}_{M}, \mathrm{ER}_{I}\right)$ and insertion losses $\left(\mathrm{IL}_{M}, \mathrm{IL}_{I}\right)$ are given in linear units.

The exact form of the ACF when the currents are sampled by a couple of ideal analog-to-digital converters (ADC) can now be immediately computed as

$$
\operatorname{ACF}\left(\beta, f_{R F}, \phi_{R F}(t)\right)=\frac{i^{\mathrm{Up}}}{i^{\text {Down }}}
$$

Although complex at first sight, the final ACF is made of mainly three types of terms. The first one $\left(I_{D C}\right)$ does not depend on the characteristics of the RF signal, but only on the parameters of the optical system. The second and third ones $\left(i_{\zeta}(\beta)\right.$ and $\left.i_{\Delta}(\beta)\right)$ are dependent on RF power through the parameter $\beta$ (phase modulation index). The last one, $i_{\Sigma}\left(\beta, f_{R F}, \phi_{R F}(t)\right)$, depends on RF power, central frequency and RF instantaneous phase, and contains the contributions from first and high-order harmonics in the signal spectrum. Under a first-order approximation most of these residual terms can be usually neglected, leading to an ACF which is essentially independent of RF power. However, as we shall see in the next section, the impact of a combination of realistic parameter values induces significant deviations in its shape. Furthermore, they introduce a non-negligible RF power dependence of the ACF curve that should be carefully taken into account in the design stage of any photonics-assisted IFM system based on this particular technique.

\section{Simulation RESUlTS AND DISCUSSION}

Thanks to the developed theoretical analysis, it is now easy to quantitatively assess the influence of each parameter on system performance. As it has been stated, the core of this particular IFM technique relies on establishing a direct one-to-one relationship between the ratio of two detected optical powers (quasi-DC photocurrents) and the instantaneous frequency of an arbitrary RF signal. In order for the system to yield accurate results, this mapping must be unique and independent of any other characteristic of the RF signal (such as power), which means that the ACF should not vary for RF pulses of different amplitudes. Furthermore, the exact shape of the ACF must be precisely known. Otherwise, systematic bias errors are introduced in the instantaneous frequency recovery process. Under perfect system operation, the measured ACF should fit a curve of the form:

$$
\operatorname{ACF}(f(t))=\frac{1+\cos (2 \pi f(t) / \mathrm{FSR})}{1-\cos (2 \pi f(t) / \mathrm{FSR})}
$$

In practice, realistic parameter values in the optical system (such as finite extinction ratios in both the MZI and MZM), significantly deviate the measured ACF from its ideal response. Thus, insight into the relative impact of each individual parameter can be gained by just looking at how much the non-ideal ACF deviates from its perfect response when these parameters fluctuate around their ideal values. Higher ACF deviations for similar parameter fluctuations will mean higher system sensitivity, helping us to determine the most critical ones. From now on, we will assume for simplicity that the input signal is not chirped, so that $f(t)=f=f_{R F}$.

\section{A. Impact of individual parameters in system performance}

As a first example, consider the effect of a non-zero phase error due to imperfect operation of the bias controllers in both the MZI and the MZM. In the MZI case (Fig. 5a), this phase error induces a wavelength offset between the MZI spectral transfer function and the optical carrier. As a result, the ACF shape changes, since the optical attenuations seen by the first-order sidebands as the RF frequency is being swept are now different. For example, a value of $\phi_{I}^{e}=90^{\circ}$ would yield a completely flat ACF. This is due to the fact that the optical carrier would be now situated at the quadrature bias point of the MZI, where the attenuation induced by the upper/lower MZI outputs at different RF frequencies is exactly the same, independently of modulation frequency. A value of $\phi_{I}^{e}=180^{\circ}$ would be equivalent to computing the inverse ratio $\left(i^{\text {Down }} / i^{\mathrm{Up}}\right)$, which is an horizontally mirrored image of the original ACF. In the MZM case (Fig. 5b), the existence of a bias phase error leads to a non-infinite suppression of the optical carrier, even if $\mathrm{ER}_{M}=+\infty$. This is due to the fact that the upper/lower branch of the MZM are no longer perfectly ouf ot phase. Now the ACF is modified asymetrically, which can be easily understood by noting that there exists a contribution to the total photocurrent due to the residual optical carrier. In the lower branch (minimum transmission point of the MZI) this residual carrier is completely suppressed since it is located at the MZI notch, and thus does not affect $i^{\text {Down }}$. However, in the upper branch (maximum transmission point of the MZI) the relative contribution of this term to $i^{\mathrm{Up}}$ gets higher as the first-order optical sidebands get more and more attenuated (higher normalized frequencies). Thus, the ACF gets more distorted at higher normalized frequencies, as it is shown in Fig. 5b. Also note that this effect is very sensitive in the MZM case. Phase deviations of a few degrees $\left(2^{\circ}, 4^{\circ}\right)$ yield the same amount of distortion as deviations of tens of degrees $\left(18^{\circ}, 36^{\circ}\right)$ in the MZI.

Next, the impact of a non-infinite ER in the Mach-Zehnders can be examined. For the MZI case, it is well-known that this implies an upper bound on the ratio between the maximum and minimum transmission points of the transfer function. Thus, a finite ER yields an upper/lower bound on the ACF, which in fact occurs when the first-order sidebands are located at either the notch of the lower branch $\left(f_{R F}=0\right)$ or at the notch of the upper branch $\left(f_{R F}=F S R / 2\right)$. As a result, the ACF symmetrically deviates from the ideal one, and its maximum/minimum values match the ER of the MZI (see Fig. 5c). In the MZM (Fig. 5d), the effect of a finite ER is essentially equal to that of a bias phase error: Because of the imperfect amplitude imbalance in the MZM arms, the optical carrier is not perfectly suppressed, and this basically leads to an asymmetrical deviation of the ACF due to the same reasons as those mentioned in the previous paragraph. Again, note that the impact of a residual optical carrier is significantly higher than that of a finite ER in the MZI. Similar ACF deviations are obtained in the MZM case for extinction ratios that are $20 \mathrm{~dB}$ higher than those in the MZI.

Last, it is also important to analyze how and why the ACF shape changes for different input RF power levels. As an 


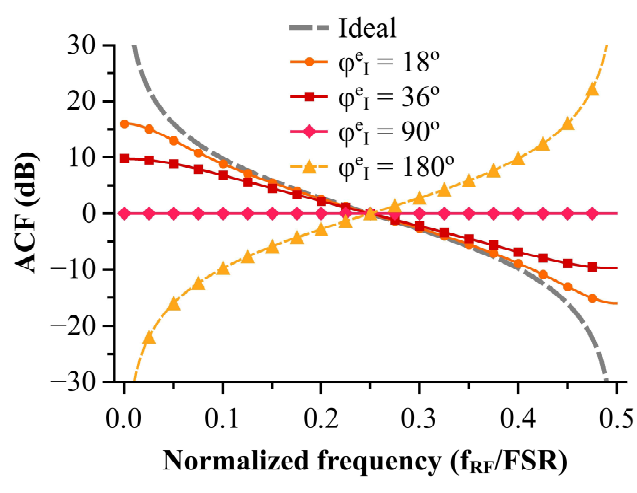

(a)

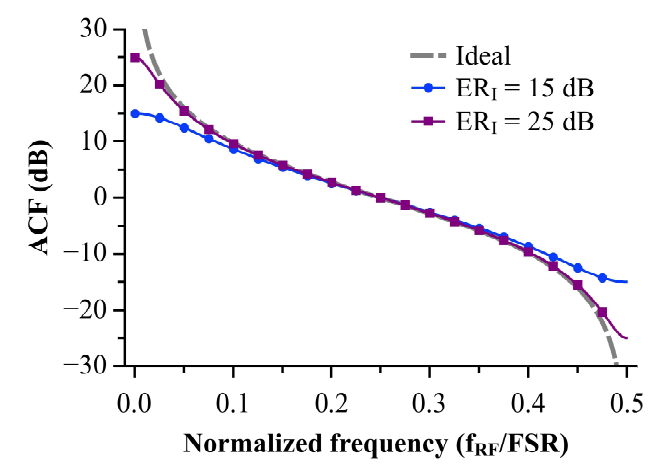

(c)

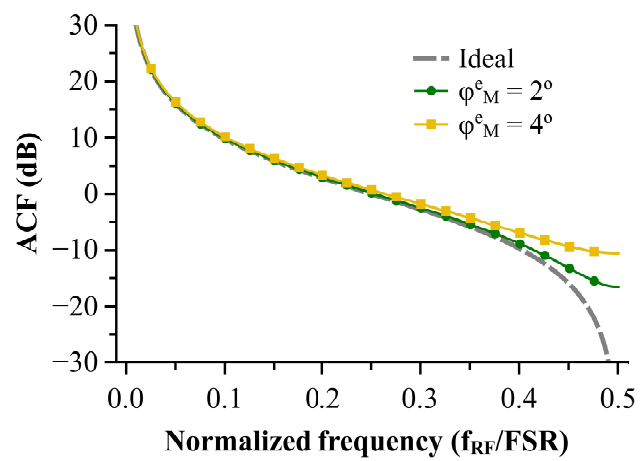

(b)

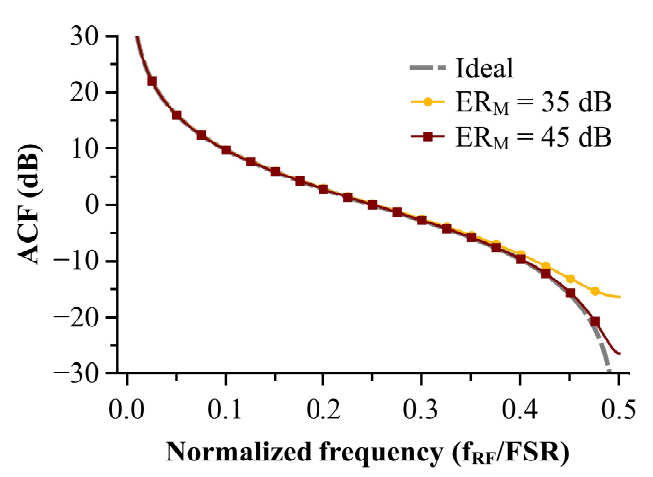

(d)

Fig. 5. Simulated ACFs for different system imperfections. (a) Bias phase error in the MZI. (b) Bias phase error in the MZM. (c) Finite extinction ratio in the MZI. (d) Finite extinction ratio in the MZM. Simulation values for all four figures are: $\mathrm{Z}_{\text {in }}=50 \Omega, \mathrm{P}_{R F}=0 \mathrm{dBm}, V_{\pi}^{A C}=3 \mathrm{~V}, \mathrm{P}_{o}=20 \mathrm{~mW}$ and $\mathcal{R}=0.9 \mathrm{~A} / \mathrm{W}$. All other parameters, except that under analysis, are assumed ideal.

example, two extreme situations are plotted in Fig. 6. Fig. 6a illustrates the effects of an extremely high input RF power, while Fig. $6 \mathrm{~b}$ shows the effect of very low powers. In the first case, the ACF gets considerably distorted because of the high value of the phase modulation index $(\beta)$. At this point, the contributions to the total photocurrent due to the high-order harmonics $\left(J_{2}^{2}(\beta), J_{3}^{2}(\beta) \ldots\right)$ dominate over the first-order ones $\left(J_{1}^{2}(\beta)\right)$, which is a direct consequence of the behaviour of the Bessel functions at high $\beta$ values. For illustration purposes, the contribution of these terms to the upper photocurrent is shown in Fig. 7 for an RF power of $+25 \mathrm{dBm}$. It can be seen that the third-order term is already significant. Only odd orders $(n=1,3,5)$ are shown because, under ideal conditions, all even order terms are zero. In the second case (Fig. 6b), the amplitude of the first-order sidebands goes to zero as $\beta$ decreases. Neglecting all other system parameters, this implies that the relative contribution of this term to the total photocurrent decreases as compared to that of the dark current, which is constant and does not depend on RF power. As a result, the ACF gets flatter and flatter as the RF power decreases, and the ratio approaches that of the two residual dark currents, which is $1(0 \mathrm{~dB})$ assuming that they are equal. These two examples show that, even considering almost perfect operation, there exists an optimum RF power at which the ACF is closest to the ideal response.

\section{B. Combined impact of parameters in system performance}

So far, all simulations have considered only one parameter at time. While this served our purpose of understanding their individual impact, it does not provide a complete vision on the interaction and combined effect of all of them at the same time. Consequently, we now consider a more realistic situation, where reasonable values for all system parameters based on state-of-the-art devices and integration technologies are included. These can be found in Table I along with appropriate references, when needed. Simulation results are plotted in Fig. 8. Fig. 8a shows the evolution of the measured ACF over the whole frequency range ([0-FSR/2]) when the input power varies between $+10 \mathrm{dBm}$ and $-20 \mathrm{dBm}$, a typical range for commercial, electronics-based IFM systems (which normally reach down to $-50 \mathrm{dBm}[32])$. It can be seen that the ACF at $+10 \mathrm{dBm}$ is near the optimum point mentioned in the previous paragraph, where just a small deviation at both high and low frequencies is observed due to optical system imperfections (phase errors, amplitude imbalance, etc.). When the power is reduced, though, the ACF flattens and asymptotically tends to a constant value of $+25 \mathrm{~dB}$. This can be understood by noting that the photocurrents generated by the residual optical carrier 


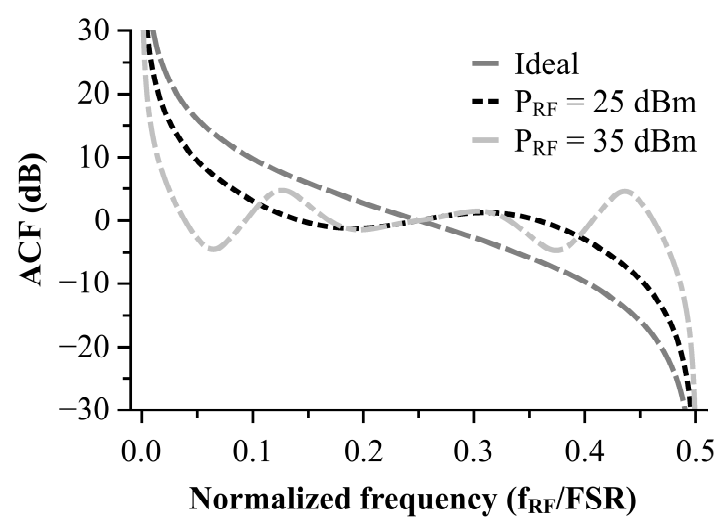

(a)

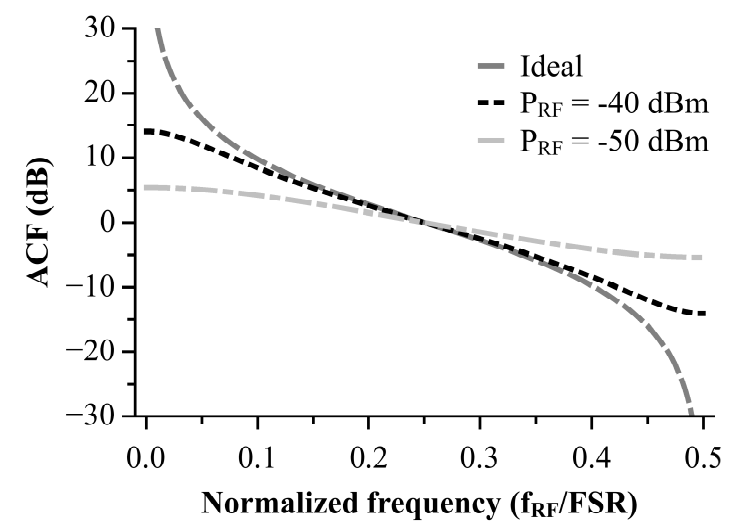

(b)

Fig. 6. Effect of (a) high powers and (b) low powers in ACF. Simulation values are: $\mathrm{Z}_{i n}=50 \Omega, V_{\pi}^{A C}=3 \mathrm{~V}, \mathrm{P}_{o}=20 \mathrm{~mW}, \mathcal{R}=0.9 \mathrm{~A} / \mathrm{W}$ and $I_{\text {Dark }}=1 \mathrm{nA}$. All other parameters are ideal.

now dominate over the dark current contribution, which is fairly small in state-of-the-art, low-speed InGaAs photodiodes. As a consequence, when RF power is very low the ACF approximates the extinction ratio of the MZI $(25 \mathrm{~dB}$ in this case), which is in fact the optical power ratio between the carrier at the maximum transmission point (MZI upper branch) and the minimum transmission point (MZI lower branch).

It can also be observed that in all cases the maximum deviation from the ideal response takes place at high frequencies. Thus, if no calibration curves are employed and Eq. (28) is directly used to estimate the input instantaneous frequency, the error introduced by this distortion will reach its maximum at FSR/2. In other words: When an RF signal with $f=\mathrm{FSR} / 2$ enters the IFM system, the normalized bias error of the measurement process (defined as $f_{e}=$ $|\hat{f}-f| / F S R)$ is maximum. This value provides an upper limit on performance and can be easily computed as follows. First, the non-ideal photocurrent ratio when $f=\mathrm{FSR} / 2$ is computed using Eq. (27) $(A C F(\beta, F S R / 2,0))$. Afterwards, this ratio is inserted into Eq. (28), which can be solved to get an estimate

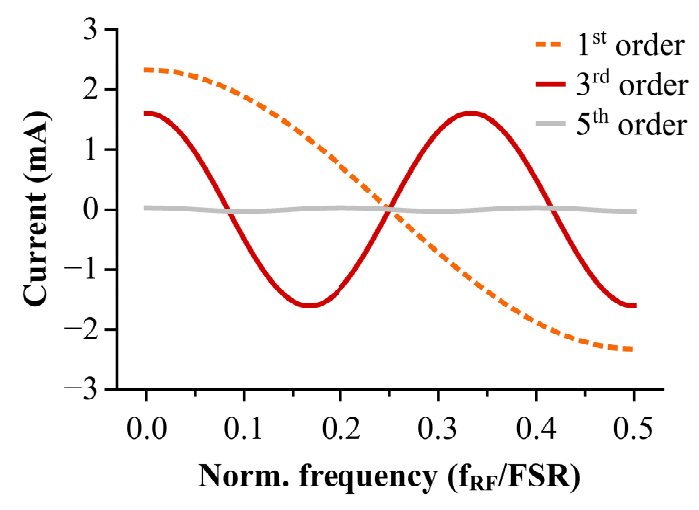

Fig. 7. Contribution of different order harmonic terms $(n=1,3,5)$ to the upper photocurrent $\left(i^{\text {up }}\right)$. Simulation values are the same of those of Fig. 6a, with $\mathrm{P}_{R F}=+25 \mathrm{dBm}$. of the instantaneous frequency $(\hat{f})$. Finally, the difference between this estimate and 0.5 (normalized frequency when $f=\mathrm{FSR} / 2$ ) is calculated, which gives the normalized bias error $\left(f_{e}\right)$. The whole process is described by the equation below:

$$
f_{e}=\left|\frac{1}{2 \pi} \arccos \left(\frac{(\mathrm{ACF}(\beta, F S R / 2,0)-1)}{(\operatorname{ACF}(\beta, F S R / 2,0)+1)}\right)-0.5\right|
$$

The dependence of $f_{e}$ versus RF power is shown in Fig. 8b for three different values of both $\mathrm{ER}_{M}$ and $\mathrm{ER}_{I}$. At high RF powers, the ACF is closest to the optimum point, and a normalized error below 0.05 is obtained for the three cases. As RF power goes below $0 \mathrm{dBm}$, the bias error rapidly increases until it reaches the maximum possible value (0.5), due to the fast evolution of the ACF as the first-order sidebands approach the residual optical carrier level. The threshold at which this happens is lower for higher extinction ratios, though errors are still significant over the whole power range.

From the previous simulations it can be concluded that, even when using state-of-the-art equipment, stringent limitations exist on the dynamic range of this photonics-assisted IFM technique. As a first approach to solve this issue, a calibration procedure at a fixed RF power is usually employed [24]. This basically entails characterizing the ACF after fabrication and then embedding it into the system hardware. Afterwards, the parametrized curve is used during the estimation process instead of Eq. (28), which eliminates the non-zero bias error that exists even near the optimum working point. However, this approach only yields accurate results over a limited RF power range, as the ACF significantly worsens at low RF powers and thus the parametrized curve is no longer valid. In that case, multiple ACF calibration curves measured at different input RF powers would be needed. This approach would in turn require a way to quickly measure the RF power of the input pulse previous to the estimation process, and then choosing the curve that was calibrated at a similar RF power. Another option, that could even be used in combination with the previous ones, would be to add a limiting amplifier at 


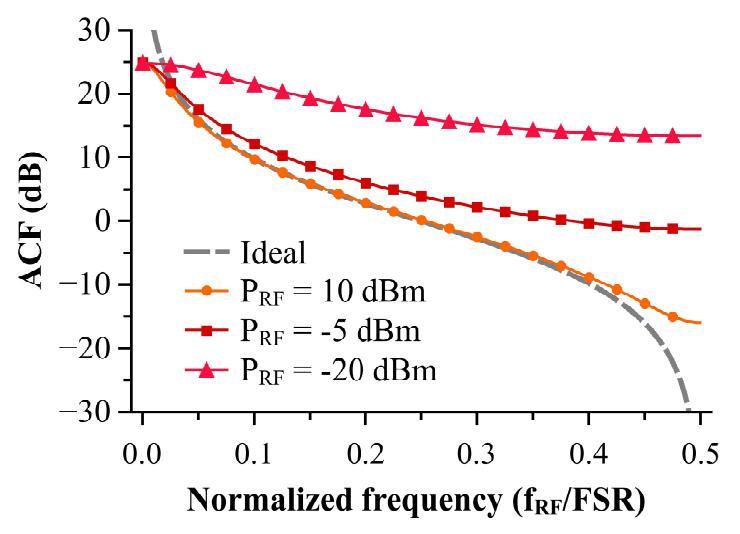

(a)

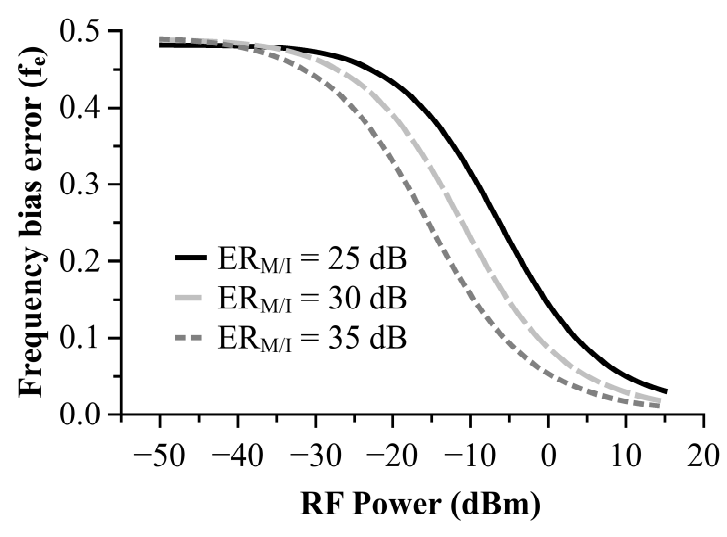

(b)

Fig. 8. (a) Evolution of ACF with different RF powers. (b) Normalized frequency bias error versus input RF power, assuming different values of both ER ${ }_{M}$ and $\mathrm{ER}_{I}$. All other simulation parameters are listed in Table I.

the input RF stage. Limiting amplifiers basically provide a constant RF power signal at their output even if the input power fluctuates over a wide dynamic range. In fact, they are commonly employed in electronic-based IFM receivers [3]. With an auxiliary limiting amplifier, the system could be optimized in such a way that the output RF power of the amplifier would be near the optimum working point of the photonic IFM system, thus minimizing error degradation over a wider dynamic range. In any case, complexity and cost would be added to the final solution.

\section{CONCLUSION}

We have developed a time domain analytical model for the analysis of system imperfections in a photonics-assisted IFM technique based on a DSB-SC optical modulation and a MZI filter. Unlike previous works, our model considers all major optical and electrical effects, such as amplitude imbalance and phase errors in the MZM and the MZI, as well as dark currents in the photodiodes. Furthermore, it includes the effect of phase/frequency modulation in the RF pulse, which is an essential feature for the analysis of modern

TABLE I

SiMULATION PARAMETERS

\begin{tabular}{cccc}
\hline Name & Value & Units & References \\
\hline$Z_{i n}$ & 50 & $\Omega$ & \\
$V_{\pi}^{A C}$ & 3 & $\mathrm{~V}$ & {$[28],[29]$} \\
$\mathrm{IL}_{M}$ & 5 & $\mathrm{~dB}$ & {$[29]$} \\
$\mathrm{ER}_{M}$ & 25 & $\mathrm{~dB}$ & {$[29]$} \\
$\phi_{M}^{e}$ & $\pm 1^{\circ}$ & degrees & {$[30]$} \\
$\mathrm{IL}_{I}$ & 5 & $\mathrm{~dB}$ & \\
$\mathrm{ER}_{I}$ & 25 & $\mathrm{~dB}$ & \\
$\phi_{I}^{e}$ & $\pm 1^{\circ}$ & degrees & {$[30]$} \\
$P_{o}$ & 20 & $\mathrm{~mW}$ & \\
$\mathcal{R}$ & 0.9 & $\mathrm{~A} / \mathrm{W}$ & {$[33]$} \\
$I_{\text {Dark }}$ & 1 & $\mathrm{nA}$ & {$[33]$} \\
\hline \multicolumn{4}{c}{}
\end{tabular}

radar signals. Numerical simulations indicate that a finite carrier suppression arising mainly from imperfections in the MZM leads to significant degradation of the IFM system performance. In particular, it has been shown that both the extinction ratio and the bias phase error in the MZM are the most critical parameters for achieving a low frequency error. Our simulations also demonstrate that the system ACF strongly depends on input RF power, thus leading to a poor performance in terms of dynamic range even when using parameter values from state-of-the-art devices. Overall, our results suggest that a careful analysis and assessment of the impact of RF power needs to be done when designing and reporting on the performance of photonics-based IFM systems. Finally, some ideas for alleviating this problem have been discussed, such as the combined use of calibration curves and limiting amplifiers in the input RF stage.

\section{REFERENCES}

[1] P. W. East, "Design techniques and performance of digital IFM," IEE Proceedings F (Commun., Radar and Signal Process.), vol. 129, no. 3, pp. 154-163, Jun. 1982.

[2] H. Gruchala and M. Czyzewski, "The instantaneous frequency measurement receiver in the complex electromagnetic environment," in Int. Conf. on Microw., Radar and Wireless Commun (MIKON), vol. 1, May 2004, pp. $155-158$.

[3] G.-C. Liang, C.-F. Shih, R. Withers, B. Cole, and M. Johansson, "Spacequalified superconductive digital instantaneous frequency measurement subsystem," IEEE Trans. Microw. Theory Techn., vol. 44, no. 7, pp 1289-1299, Jul. 1996.

[4] M. de Souza, F. Silva, M. De Melo, and L. R. G. S. L. Novo, "Discriminators for instantaneous frequency measurement subsystem based on open-loop resonators," IEEE Trans. Microw. Theory Techn., vol. 57, no. 9, pp. 2224-2231, Sep. 2009.

[5] L. Nguyen and D. Hunter, "A photonic technique for microwave frequency measurement," IEEE Photon. Technol. Lett., vol. 18, no. 10, pp. 1188-1190, May 2006.

[6] H. Chi, X. Zou, and J. Yao, "An approach to the measurement of microwave frequency based on optical power monitoring," IEEE Photon. Technol. Lett., vol. 20, no. 14, pp. 1249-1251, Jul. 2008.

[7] X. Zou and J. Yao, "An optical approach to microwave frequency measurement with adjustable measurement range and resolution," IEEE Photon. Technol. Lett., vol. 20, no. 23, pp. 1989-1991, Dec. 2008. 
[8] H. Emami, N. Sarkhosh, L. A. Bui, and A. Mitchell, "Amplitude independent RF instantaneous frequency measurement system using photonic Hilbert transform," Opt. Express, vol. 16, no. 18, pp. $13707-$ 13712 , Sep. 2008.

[9] M. V. Drummond, P. Monteiro, and R. N. Nogueira, "Photonic RF instantaneous frequency measurement system by means of a polarizationdomain interferometer," Opt. Express, vol. 17, no. 7, pp. 5433-5438, Mar. 2009.

[10] J. Zhou, S. Fu, S. Aditya, P. Shum, and C. Lin, "Instantaneous microwave frequency measurement using photonic technique," IEEE Photon. Technol. Lett., vol. 21, no. 15, pp. 1069-1071, Aug. 2009.

[11] H. Guo, G. Xiao, N. Mrad, and J. Yao, "Measurement of microwave frequency using a monolithically integrated scannable Echelle diffractive grating," IEEE Photon. Technol. Lett., vol. 21, no. 1, pp. 45-47, Jan. 2009.

[12] X. Zou, H. Chi, and J. Yao, "Microwave frequency measurement based on optical power monitoring using a complementary optical filter pair,' IEEE Trans. Microw. Theory Techn., vol. 57, no. 2, pp. 505-511, Feb. 2009.

[13] S. Pan and J. Yao, "Instantaneous microwave frequency measurement using a photonic microwave filter pair," IEEE Photon. Technol. Lett., vol. 22 , no. 19 , pp. 1437-1439, Oct. 2010.

[14] X. Zou, W. Pan, B. Luo, and L. Yan, "Photonic instantaneous frequency measurement using a single laser source and two quadrature optical filters," IEEE Photon. Technol. Lett., vol. 23, no. 1, pp. 39-41, Jan. 2011.

[15] S. Pan, J. Fu, and J. Yao, "Photonic approach to the simultaneous measurement of the frequency, amplitude, pulse width, and time of arrival of a microwave signal," Opt. Lett., vol. 37, no. 1, pp. 7-9, Jan. 2012.

[16] W. Li, N. H. Zhu, and L. X. Wang, "Brillouin-assisted microwave frequency measurement with adjustable measurement range and resolution," Opt. Lett., vol. 37, no. 2, pp. 166-168, Jan. 2012.

[17] S. Zheng, S. Ge, X. Zhang, H. Chi, and X. Jin, "High-resolution multiple microwave frequency measurement based on stimulated Brillouin scattering," IEEE Photon. Technol. Lett., vol. 24, no. 13, pp. 1115-1117, Jul. 2012 .

[18] D. Marpaung, "On-chip photonic-assisted instantaneous microwave frequency measurement system," IEEE Photon. Technol. Lett., vol. 25, no. 9, pp. 837-840, May 2013.

[19] L. A. Bui and A. Mitchell, "Remoted all optical instantaneous frequency measurement system using nonlinear mixing in highly nonlinear optical fiber," Opt. Express, vol. 21, no. 7, pp. 8550-8557, Apr. 2013.

[20] B. Lu, W. Pan, X. Zou, B. Luo, L. Yan, X. Liu, and S. Xiang, "Photonic frequency measurement and signal separation for pulsed/CW microwave signals," IEEE Photon. Technol. Lett., vol. 25, no. 5, pp. 500-503, Mar. 2013.

[21] M. S. et al., "An introduction to InP-based generic integration technology," Semiconductor Science and Technology, vol. 29, no. 8, p. 083001, 2014.

[22] M. Heck, J. Bauters, M. Davenport, J. Doylend, S. Jain, G. Kurczveil, S. Srinivasan, Y. Tang, and J. Bowers, "Hybrid silicon photonic integrated circuit technology," IEEE J. Sel. Topics Quantum Electron., vol. 19, no. 4, pp. $6100117-6100117$, Jul. 2013.

[23] D. Marpaung, C. Roeloffzen, R. Heideman, A. Leinse, S. Sales, and J. Capmany, "Integrated microwave photonics," Laser \& Photonics Reviews, vol. 7, no. 4, pp. 506-538, 2013.

[24] J. S. Fandiño and P. Muñoz, "Photonics-based microwave frequency measurement using a double-sideband suppressed-carrier modulation and an InP integrated ring-assisted Mach-Zehnder interferometer filter," Opt. Lett., vol. 38, no. 21, pp. 4316-4319, Nov. 2013.

[25] S. Harmon, V. Urick, J. Diehl, and K. Williams, "Tandem electrooptic modulation and interferometric detection: Theory and application," IEEE Photon. Journal, vol. 5, no. 4, pp. 5 501 211-5 501 211, Aug. 2013.

[26] P. Muñoz, J. Capmany, D. Perez, J. H. den Besten, J. S. Fandiño, and D. Domenech, "Integrated microwave photonics: State of the art and future trends," in Int. Conf. on Transparent Opt. Netw. (ICTON), Jul. 2014.

[27] E. Wooten, K. Kissa, A. Yi-Yan, E. Murphy, D. Lafaw, P. Hallemeier, D. Maack, D. Attanasio, D. Fritz, G. McBrien, and D. Bossi, "A review of lithium niobate modulators for fiber-optic communications systems," IEEE J. Sel. Topics Quantum Electron., vol. 6, no. 1, pp. 69-82, Jan. 2000.

[28] R. Griffin, S. Jones, N. Whitbread, S. Heck, and L. Langley, "InP MachZehnder modulator platform for 10/40/100/200-Gb/s operation," IEEE J. Sel. Topics Quantum Electron., vol. 19, no. 6, pp. 158-166, Nov. 2013.
[29] K. Prosyk, A. Ait-Ouali, C. Bornholdt, T. Brast, M. Gruner, M. Hamacher, D. Hoffmann, R. Kaiser, R. Millett, K. Velthaus, and I. Woods, "High performance $40 \mathrm{GHz}$ InP Mach-Zehnder modulator," in Optical Fiber Communication Conference (OFC/NFOEC), Mar. 2012, pp. 1-3.

[30] [Online]. Available: http://www.photonicsinc.com/modulator_bias_ controller.html

[31] P. Besse, M. Bachmann, H. Melchior, L. Soldano, and M. Smit, "Optical bandwidth and fabrication tolerances of multimode interference couplers," J. Lightw. Technol., vol. 12, no. 6, pp. 1004 -1009, Jun. 1994.

[32] [Online]. Available: http://www.widebandsystems.com/ifm.html

[33] [Online]. Available: http://www.hamamatsu.com/eu/en/index.html

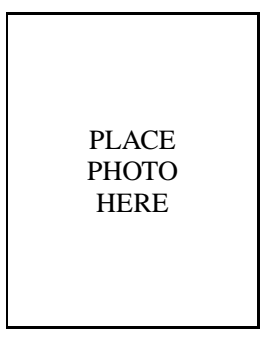

Javier S. Fandiño was born in Orihuela (Alicante), in 1987. He received his bachelor degree in Telecommunications engineering and the M.S. degree in Technology, Systems and Telecommunications Networks, both from the Universitat Politècnica de València (Spain), in 2010 and 2011, respectively. In 2011, he joined the Optical and Quantum Communications Group (OQCG), where he was first engaged in the experimental demonstration of an alloptical 2R regenerator for extended PON networks. At present, he is pursuing his $\mathrm{PhD}$ in Telecommunications engineering in the OQCG, supported by the Spanish Ministry of Science and Innovation trough a FPU (Formación de Personal Universitario) scholarship. His research topics include the design and characterization of photonic integrated circuits in different technologies (such as Silicon-OnInsulator and InGaAsP), and their potential application to microwave photonic systems.

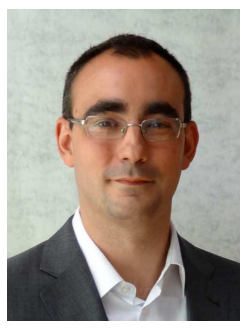

Pascual Muñoz was born in València, Spain on February 7th, 1975. He received his Ingeniero de Telecomunicación degree from Universitat Politècnica de València (UPV, Spain) in 1998. In 1999 he served as 1st Lieutenant in the Spanish Airforce, while working as IT consultant for AIME Instituto Tecnológico. He received a Ph.D. degree in photonics from UPV in 2003. He is currently Associate Professor at the Departamento de Comunicaciones, and researcher at the Instituto de Telecomunicaciones y Aplicaciones Multimedia (ITEAM), both at UPV. Dr. Muñoz runs a consolidated research line, started in 2005, on prototyping Photonic Integrated Circuits (PICs) in a technology agnostic fashion, where PICs are designed in the best suited technology (Silicon-OnInsulator, Indium Phosphide, Silica on Silicon, Silicon Nitride amongst other) for each application. He has been involved in several European Commission funded projects, being coordinator for integration on InP within the NoE IST-EPIXNET. He has published over 25 papers in international refereed journals and over 40 conference contributions. He is a member of the Technical Programme Committees of the European Conference on Optical Communications (ECOC) and the European Conference on Integrated Optics (ECIO). Dr. Muñoz received the VPI Speed Up Photonics Award in 2002 for innovative Fourier optics AWG with multimode interference (MMI) couplers modeling, by Virtual Photonics Incorporated and IEEE Communications Magazine. He was also granted the IEEE/LEOS Graduate Student Fellowship Program in 2002. He received the extraordinary doctorate prize from UPV in 2006. From his research line, he co-founded the UPV spin-off company VLC Photonics in 2011, where the PIC design know-how, expertise and tools have been transferred, and he served as CEO from 2011 to 2013. Dr. Muñoz is a Senior Member of IEEE and Member of the OSA. 